\title{
Carbon C-14
}

National Cancer Institute

\section{Source}

National Cancer Institute. Carbon C-14. NCI Thesaurus. Code C1638.

An unstable isotope of carbon created when a neutron collides with a nitrogen atom, causing capture of the neutron and release of the proton converting nitrogen to a carbon with fourteen nucleons ( 6 protons and 8 neutrons). Carbon- 14 emits beta particles as it decays and reverts back to nitrogen. 